\title{
Significance of atypical presentation of symptomatic SUNCT: a case report
}

\author{
J W M ter Berg, P J Goadsby
}

\begin{abstract}
A patient with a SUNCT-like syndrome caused by severe basilar impression in association with osteogenesis imperfecta is described. Initially symptoms of both the first and second branch of the trigeminal nerve were prominent, on which carbamazepine had only a temporary and mild effect. Progressive symptoms with prominent ipsilateral autonomic features, unexplained triggering by photostimulation, and increasing duration of pain attacks occurred with relentless progressive basilar impression associated with pontomedullary compression. Pathophysiologically a dysfunction in ephaptic transmission is hypothesised.

(F Neurol Neurosurg Psychiatry 2001;70:244-246)
\end{abstract}

Keywords: SUNCT syndrome; symptomatic; basilar impression

SUNCT syndrome is a unusual form of primary headache, characterised by attacks of short lasting, unilateral pain of a neuralgiform character, accompanied by prominent conjunctival injection and tearing and rhinorrhoea. ${ }^{1}$ It is among the rarest of the headache syndromes. ${ }^{2}{ }^{3}$ To the best of our knowledge, 33 cases have been described. It has been proposed that SUNCT syndrome is classified with other primary headaches with more or less prominent autonomic features - such as cluster headache, chronic paroxysmal hemicrania $(\mathrm{CPH})$, episodic paroxysmal hemicrania $(\mathrm{EPH})$, hemicrania continua, and the clustertic syndrome - as trigeminal-autonomic cephalalgias. ${ }^{3}$ This terminology recognises the physiology of the trigeminal-autonomic vasodilator reflex ${ }^{4}$ which has as its afferent the ophthalmic (first) division of the trigeminal nerve and as its efferent the cranial parasympathetic outflow through the VIIth (facial) nerve.

Clinical descriptions of SUNCT suggest a pain duration of between 5-250 seconds and frequencies of one attack/day to 30 attacks in 1 hour. The orbitotemporal pain localisation in SUNCT is in the area of the first branch of the trigeminal nerve. In some cases the differentiation between SUNCT and trigeminal neuralgia may be difficult ${ }^{5}$ and a case with transformation of trigeminal neuralgia into SUNCT syndrome is illustrative of a possible con- tinuum. ${ }^{6}$ There are three reported cases of symptomatic SUNCT which have in common a posterior fossa lesion ${ }^{7-9}$ which may have implications for understanding SUNCT. In this report we present a new symptomatic case with clinical features resembling SUNCT, again with pathology in the posterior fossa.

\section{Case report}

The patient, a wheelchair bound $30 \mathrm{~kg}$ man, height $110 \mathrm{~cm}$ and normal sclera with non-familial osteogenesis imperfecta could never walk because of severe bone fragility and progressive skeletal deformities. He had frequent spontaneous bone fractures until the age of 22 and had increasing bilateral hearing loss from age 25 . He had hypertension recognised from the age of 44 . At the age of 45 he underwent a left sided stapes interposition because of stapes ankylosis. Family history disclosed that five of nine brothers and sisters died from myocardial infarction.

At the age of 40 , the patient presented initially with attacks of intense lancinating pain in the left supraorbital and infraorbital region present for 1 day with five discrete episodes each of 100-300 attacks without autonomic symptoms, such as lacrimation. The patient was not known to have had headaches before. Brain CT disclosed a mild basilar impression, which was judged to be unrelated. The diagnosis of trigeminal neuralgia was made and treatment with carbamazepine (200 mg/day) was initiated. Symptoms did not occur until 2 years later. Carbamazepine had to be increased to $600 \mathrm{mg} /$ day because of recurrent neuralgiform pain which was now associated with ipsilateral lacrimation. Three years later he presented to one of us (HTB).

On presentation the patient had left sided sharp, excruciating pain with a duration of 2-3 minutes and a frequency of one to five times a day in association with nausea, ipsilateral lacrimation, facial flushing, conjunctival injection, and bilateral rhinorrhoea. After some months the duration increased to 30-60 minutes, and the pain quality changed to a deep excruciating, boring pain with 10-15 episodes a day of superimposed lancinating pain. There were no triggers. Neuralgiform attacks with a duration of about 1 minute recurred in association with the autonomic symptoms. The patient developed triggers of sudden exposure to light and 


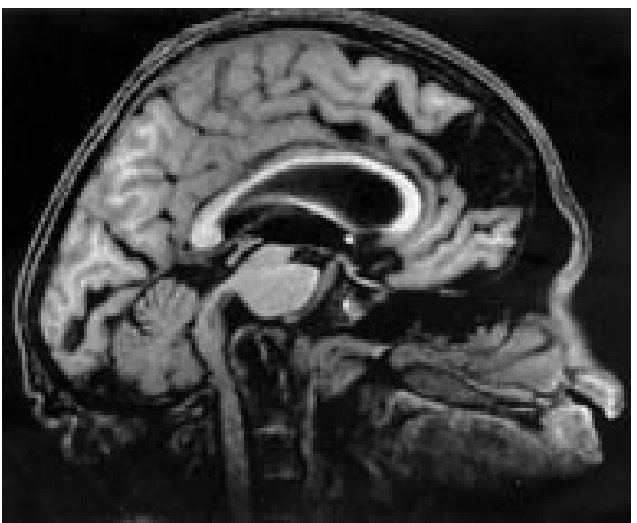

Figure 1 Midline sagittal T1 weighed 1 Tesla MRI of the cranium and craniocervical junction of the patient with osteogenesis imperfecta and symptomatic SUNCT. Note the severe platybasia with marked basilar impression and distortion of the posterior fossa anatomical relations. The odontoid process produces pronounced pontomedullary angulation Frontotemporal atrophy and ventricular enlargement are also visible.

intensive sounds, chewing, talking, and swallowing. He stayed for weeks in a dark environment because of provocation by both light exposure and photophobia. Increasing carbamazepine therapy to $1800 \mathrm{mg} /$ day had only temporary benefit. The trigger factors expanded to include upward positioning of the head and trunk which also caused vertical and oblique diplopia, symptoms that could be improved by lying down. Between attacks there was a continuous mild left sided periorbital and auricular pain with low frequency ipsilateral blepherospasm.

Repeated neurological examination was normal, particularly coordination. Brain CT and MRI of the skull showed platybasia with marked basilar impression, bilateral frontotemporal atrophy, ventricular enlargement, and marked pontomedullary angulation in association with a left sided mass between the pons and cerebellum, defined as a part of a cerebellar lobe at the level of the trigeminal and facial nerve (fig 1). Blink reflex studies disclosed an absent left sided R1 response with left sided masseter reflex, and a normal right sided R1 and bilateral $\mathrm{R} 2$ response, indicating a V-VII lesion. Brain stem auditory evoked potential was inconclusive because of the inner ear lesions. Visual evoked potential was normal.

A tentative diagnosis of symptomatic atypical SUNCT syndrome was made. During his illness he was treated with carbamazepine (up to $1800 \mathrm{mg} /$ day), phenytoin (200 mg/day), verapamil (120 mg/day), sumatriptan (6 mg subcutaneously), amitriptyline (50 mg/day), indomethacin ( $75 \mathrm{mg} /$ day), lidocaine (4\% intranasally), methysergide ( $3 \mathrm{mg} /$ day), valproate (900 mg/day), and also electrocoagulation of the pterygopalatine ganglion. Trigeminal thermocoagulation was not possible for anatomical reasons. Operative treatment was considered but the patient thought the operative risks too great. None of the treatments resulted in acceptable pain reduction except carbamazepine in the early phase of the illness. The patient died when nearly 50 years old because of coronary heart disease, ascertained

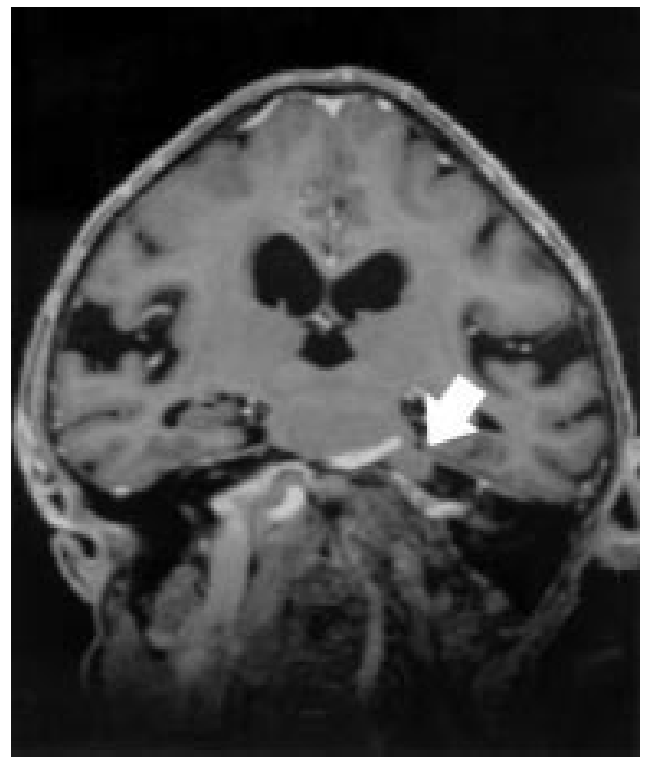

Figure 2 Coronal T1 weighed MRI of the same patient showing a mass which is a part of the cerebellum located at the left side of the pons (see white arrow).

by necropsy, which also confirmed the known macroscopical intracranial deformations (fig 2). No abnormalities could be found by microscopy in the area of the posterior structures and the trigeminal system on the pontomesencephalic level.

\section{Discussion}

The case is remarkable for both the symptoms and the possible mechanisms of the presentation of a SUNCT-like syndrome. We diagnosed this case as SUNCT-like syndrome because of the many short lasting attacks and the prominent autonomic symptoms. The modest but present effect of carbamazepine and the nature of the patient's cranial anatomy with the burden of abnormality falling on the posterior fossa, are both interesting aspects of the case.

The initial symptoms of this patient were similar to trigeminal neuralgia although it did involve both the first and second trigeminal divisions and the episodic pain duration increased up to 2-3 minutes, which would be unusual in typical trigeminal neuralgia. This overlap is somewhat similar to the cluster-tic syndrome. ${ }^{10}$ The triggering of pain attacks by photostimulation is noteworthy and there is no obvious explanation. Prominent ipsilateral autonomic features, which are an essential component of SUNCT, do occur in the course of this patient's disease as the symptoms evolve. The late occurrence of triggers and autonomic features may be caused by the relentless progressive basilar impression and associated pontomedullary compression.

Osteogenesis imperfecta is a heterogeneous disorder of connective tissue. On clinical grounds the patient could be classified as type III, because of the very severe progressive deformity of the long bones and spine. ${ }^{11}$ The adult onset conductive hearing loss is more compatible with type I, which has less bone deformity. However, some overlap between clinical types may occur. ${ }^{12}$ Neurological com- 
plications of osteogenesis imperfecta include basilar impression with brainstem compression, macrocephaly, cortical atrophy, increased intracranial pressure caused by hydrocephalus, ${ }^{12}$ and trigeminal neuralgia. ${ }^{13}$ Pathophysiological mechanisms associated with basilar impression may include impaired CSF outflow, deformation of the skull base, forces generated by neck muscles to keep the head upright, and venous outflow obstruction through the skull base. ${ }^{12}$

The association of short lived unilateral pain and ipsilateral autonomic features in the primary headache disorders, such as cluster headache, paroxysmal hemicrania, SUNCT, and cluster-tic syndrome was recently classified as trigeminal autonomic cephalgias (TACs). ${ }^{3}$ In our patient without any neurological feature with exception of SUNCT, both compression and traction forces at the level of the brainstem may have been relevant to the clinical presentation. It is remarkable that the three known symptomatic patients presented with long standing cerebellopontine angle pathology, an arteriovenous malformation, ${ }^{7}$ cavernous hemangioma in the anterolateral part of the pons in close proximity to the trigeminal nerve root entry zone, ${ }^{9}$ and a venous angioma described as being in the region of the brainstem and right cerebellar hemisphere. ${ }^{8}$ In our patient there was a longstanding gradually progressive parenchymal distortion by the basilar impression which subsequently may have caused an ephaptic transmission disturbance. It could be suggested that this alone, or more likely, in combination with an underlying genetic predisposition, is the likely basis for the headache syndrome The initial transient effect of carbamazepine supports the relation with trigeminal neuralgia and has been also reported in two other symptomatic SUNCT cases. ${ }^{67}$
In summary, a case of secondary, symptomatic, SUNCT syndrome in a patient with osteogenesis imperfecta is reported. The posterior fossa abnormalities emphasise the absolute need for MRI in any suspected case of SUNCT as all symptomatic patients have had findings in the posterior fossa. SUNCT in all its forms, but particularly when there are overlaps with trigeminal neuralgia at some clinical level, underscores the importance of the trigeminal autonomic reflex and its role in primary headache syndromes.

1 Sjaastad O, Saunte C, Salvesen R, et al. Shortlasting unilateral neuralgiform headache attacks with conjunctival injection, tearing, sweating, and rhinorrhea. Cephalalgia tion, tearing,

2 Pareja JA, Sjaastad O. SUNCT syndrome. A clinical review. Headache 1997;37:195-202.

3 Goadsby PJ, Lipton RB. A review of paroxysmal hemicranias, SUNCT syndrome and other short-lasting headaches with autonomic features, including new cases. Brain 1997; 120:193-209.

4 May A, Goadsby PJ. The trigeminovascular system in humans: pathophysiological implications for primary headache syndromes of the neural influences on the cerebral circulation. F Cereb Blood Flow Metab 1999;19:115-27.

5 Benoliel R, Sharav Y. Trigeminal neuralgia with lacrimation or SUNCT syndrome? Cephalalgia 1998;18:85-90.

6 Bouhassira D, Attal N, Esteve M, et al. SUNCT syndrome. Bouhassira $\mathrm{D}$, Attal $\mathrm{N}$, Esteve $\mathrm{M}$, et al. SUNCT syndrome.
A case of transformation from trigeminal neuralgia. CephaA case of transformatio
lalgia $1994 ; \mathbf{1 4}: 168-70$.

7 Bussone G, Leone M, Volta GD, et al. Short-lasting unilateral neuralgiform headache attacks with tearing and conjunctival injection: the first symptomatic case. Cephalalgia 1991;11:123-7.

8 Morales F, Mostacero E, Marta J, et al. Vascular malformation of the cerebellopontine angle associated with SUNCT syndrome. Cephalalgia 1994;14:301-2.

9 De Benedittis G. SUNCT syndrome associated with cavernous angioma of the brain stem. Cephalalgia 1996;16: 503-6.

10 Mulleners WM, Verhagen WIM. Cluster-tic syndrome. Neurology 1996;47:302.

11 Sillence DO. Craniocervical abnormalities in osteogenesis imperfecta: genetic and molecular correlation. Pediatr Radiol 1994;24:427-30.

12 Charnas LR, Marini JC. Communicating hydrocephalus, basilar invagination, and other neurological features in basilar invagination, and other neurological featur
osteogenesis imperfecta. Neurology 1993;43:2603-8.

13 Reilly MM, Valentine AR, Ginsberg L. Trigeminal neuralgia associated with osteogenesis imperfecta. $\mathcal{F}$ Neurol Neurosurg Psychiatry 1995;58:665. 